\title{
Atomic Resolution Crystal Field Splitting Mapping in Polar Vortices Oxide Superlattices
}

Sandhya Susarla ${ }^{1}$, Sujit Das ${ }^{2}$, Weichuan Huang ${ }^{2}$, Colin Ophus ${ }^{1}$, Peter Ercius ${ }^{1}$ and Ramamoorthy Ramesh $^{2}$

${ }^{1}$ Lawrence Berkeley National Laboratory, Berkeley, California, United States, ${ }^{2}$ University of CaliforniaBerkeley, Berkeley, California, United States

Novel topological phases such as polar vortices and skyrmions in the complex oxide systems have generated enormous interest in the condensed matter physics community due to their exotic properties like negative capacitance and chiral domains. ${ }^{1-4}$ One such topological form; polar vortices have been recently identified in $\mathrm{PbTiO}_{3} / \mathrm{SrTiO}_{3}$ (PTO/STO) superlattices grown on $\mathrm{DyScO}_{3}$ (DSO) by controlling of elastic, electrostatic and gradient energy. ${ }^{2}$ The atomic structure of polar vortices have been studied using fourdimensional scanning transmission electron microscopy (4D-STEM) and reciprocal space mapping (RSM) but these techniques do not probe electronic structure changes like crystal field splitting and spinorbit coupling. ${ }^{2,5}$ To some extent, the chiral nature of the polar vortices has been probed using X-Ray circular dichroism (XCD) but the lack of spatial resolution still limits the measurement to a certain extent. ${ }^{3}$ Electron energy near edge spectroscopy (ELNES) allows us to probe the K, L and M edges of transition metals; giving information about the spin-orbit coupling and crystal field splitting. With the continuously changing polarization in vortices, ELNES provides an ideal platform to study local electronic structure changes. Measuring spin-orbit coupling and crystal field splitting requires STEM-EELS mapping at atomic resolution with extremely high signal to noise.

We have studied the crystal field splitting of Titanium (Ti) L-edge in the PTO/STO vortices grown by pulsed laser deposition. High resolution STEM-EELS mapping was performed using TEAM I at Lawrence Berkeley National Laboratory. Monochromated ( 0.2 eV FWHM) electron energy loss spectroscopy (mono-EELS) measurements were performed using a Gatan K3 camera installed in a Gatan Continuum GIF. Spectra were de-noised using principle component analysis (PCA) where the first 3 components were used. $^{6}$

Figure 1a shows the HAADF-STEM image of 15x15 unit cells of PTO/STO superlattice. Vortices in the superlattices were identified by A-Site Pb column position fitting. The B sites were not intense enough the accurately determine the displacement. However, these polar vortices also have slight A site displacement as consequence of the B site displacement. Figure $1 \mathrm{~b}$ shows the relative displacement of $\mathrm{Pb}$ atoms with respect to conventional $\mathrm{PbTiO}_{3}$. The red color indicates leftward displacement whereas the blue color indicated the rightward displacement. The core of the vortices is present at the border of red and blue regions. We can also notice the vortices region as slight dark contrast in PTO layer in the HRHAADF-STEM image shown in Figure 1a (indicated by white dashes). The vortex regions were mapped by STEM-EELS to determine the local changes in the Ti L edge. Figure 1c shows the simultaneously acquired HAADF-STEM image acquired during the EELS mapping. Figure 1d shows the PCA enhanced EELS spectra taken from the vortex, PTO and STO respectively. Ti L edge measures the delocalization of electrons in $2 \mathrm{p}_{2} / 3$ and $2 \mathrm{p}_{1 / 3}$ to $3 \mathrm{~d}$ levels as $\mathrm{L}_{3}$ and $\mathrm{L}_{2}$ transitions. In case of the $\mathrm{O}_{\mathrm{h}}$ symmetry there is crystal field splitting as $\mathrm{t}_{2 \mathrm{~g}}$ and $\mathrm{e}_{\mathrm{g}}$ due to strong spin-orbit coupling of the transition metal. Crystal field 
splitting in the Ti L edge decreases from STO to PTO as the symmetry changes from $\mathrm{O}_{\mathrm{h}}$ to $\mathrm{D}_{4 \mathrm{~h}}$. However, as we enter the vortex region, the $e_{g}$ peak of the $\mathrm{L}_{3} \mathrm{Ti}$ edge further splits into two peaks. The e $\mathrm{g}$ peak in the $\mathrm{L}_{3} \mathrm{Ti} L$ edge indicates the hybridization of $\mathrm{d}_{\mathrm{z} 2}$ and $\mathrm{d}_{\mathrm{x} 2}$-y2 orbitals of Ti with $\mathrm{O} 2 \mathrm{p}$. Usually the e $\mathrm{e}_{\mathrm{g}}$ peak is split in cases of unstrained PTO. A previous theoretical report indicates that this splitting is caused by the paraelectric nature of tetragonal PTO. ${ }^{7}$
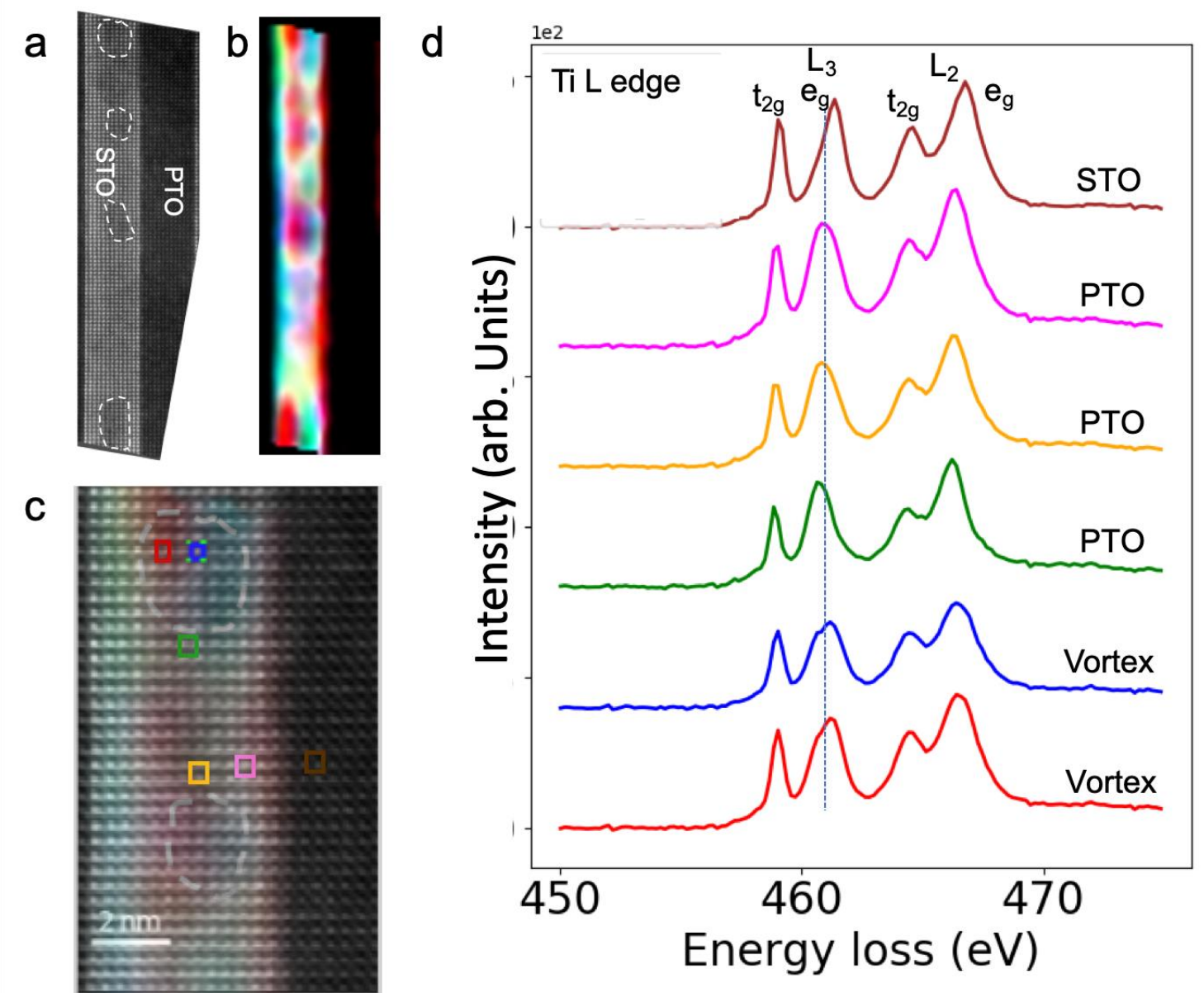

Figure 1. a) Atomic resolution drift corrected HAADF-STEM image of PTO/STO superlattice. b) A-site fitting maps showing the relative displacement of A sites in the vortices. The red color represents left shift and the blue color represents right shift. The vortices are located in between the red and blue regions. c) Simultaneously acquired HAADF-STEM image during EELS scans. The displacement maps in (b) are superimposed on the image. d) Series of EELS spectra extracted from boxes indicated in (c). Inside the vortices region, the eg peak of the Ti L edge splits into 2 peaks.

\section{References}

1. Das, S. et al. Observation of room-temperature polar skyrmions. Nature 568, 368-372 (2019).

2. Yadav, A. K. et al. Observation of polar vortices in oxide superlattices. Nature 530, 198-201 (2016).

3. Shafer, P. et al. Emergent chirality in the electric polarization texture of titanate superlattices. Proc. Natl. Acad. Sci.115, 915-920 (2018).

4. Yadav, A. K. et al. Spatially resolved steady-state negative capacitance. Nature 565, 468-471 (2019).

5. Li, Q. et al. Quantification of flexoelectricity in PbTiO 3/SrTiO 3 superlattice polar vortices using machine learning and phase-field modeling. Nat. Commun. 8, 1-8 (2017). 
6. Muller, D. A. et al. Atomic-Scale Chemical Imaging of Composition and Bonding by AberrationCorrected Microscopy. Science, 319, 1073 LP - 1076 (2008).

7. Torres-Pardo, A. et al. Spectroscopic mapping of local structural distortions in ferroelectric $\mathrm{PbTiO}$ 3/SrTiO 3 superlattices at the unit-cell scale. Phys. Rev. B 84, 220102 (2011). 